FACTA UNIVERSITATIS

Series: Mechanical Engineering Vol. 16, N² 2, 2018, pp. 115 - 126

https://doi.org/10.22190/FUME180321014C

Original scientific paper

\title{
A CRITICAL ASSESSMENT OF KASSAPOGLOU'S STATISTICAL MODEL FOR COMPOSITES FATIGUE
}

\author{
UDC 519.2:539.4
}

\author{
Michele Ciavarella $^{1}$, Giuseppe Carbone ${ }^{1}$, Vladimir Vinogradov ${ }^{2}$ \\ ${ }^{1}$ DMMM, Politecnico di BARI, Italy \\ ${ }^{2}$ School of Civil Engineering \& Geosciences, Newcastle University, U.K.
}

\begin{abstract}
Kassapoglou has recently proposed a model for fatigue of composite materials which seems to suggest that the fatigue SN curve can be fully predicted on the basis of the statistical distribution of static strengths. The original abstract writes expressions for the cycles to failure as a function of $R$ ratio are derived. These expressions do not require any curve fitting and do not involve any experimentally determined parameters. The fatigue predictions do not require any fatigue tests for calibration". These surprisingly ambitious claims and attractive results deserve careful scrutiny. We contend that the result, which originates from the reliability theory where exponential distributions is sometimes used to model distribution of failures when age (or wearout) has no influence on the probability of failure, does not conform to a fatigue testing with the resulting SN curve distribution. Despite Kassapoglou's attempt to use a wearout law which seems to confirm this result even with wearout, we contend that a proper statistical treatment of the fatigue process should not make wear-out constants disappear, and hence the SN curves would depend on them, and not just on scatter of static data. These concerns explain the large discrepancies found by 3 independent studies which have tried to apply Kassapoglou's model to composite fatigue data.
\end{abstract}

Key Words: Composite Materials, Fatigue, Wearout Models, Kassapoglou Model, Strength-life Equal Rank, Statistics

\section{INTRODUCTION}

The strength-life equal rank assumption wear-out models for fatigue of composite materials were first presented by Hahn and Kim [1], and later as a fitting approach to fatigue data by Sendeckyj, which hides a derivation based on a "damage tolerance" approach [2]. A

Received March 21, 2018 / Accepted May 12, 2018

Corresponding author: Michele Ciavarella

DMMM, Politecnico di BARI, Italy

E-mail: mciava@poliba.it 
significantly different model has been proposed more recently by Kassapoglou [3-5] (in the following, "Ref. [3-5]"), which in fact claims an extremely strong result: that of predicting SN curve of a material from just the static data. Already in the end of the 1800's for metals there were attempts to relate static data to fatigue ones, and even today only crude approximations can be made on fatigue limit over static strength (the so-called fatigue ratio), which are generally based on hardness tests. The use of simplified equations for SN curve is also well known in any fatigue textbook [6], but it is always clearly shown that any such empirical equation is limited, and that it makes, in general, only a very crude estimate. This justifies the industry of fatigue machine testing, which is by no means less flourishing in composite materials although composites are known to suffer more crucially to impact than to fatigue. Hence no aircraft flying today is there without having passed a very serious fatigue testing certification procedure, and for a good reason. Fatigue testing is required by any certification agency to get airworthiness certificates, and the cost of testing is huge. The idea to obtain even approximate results for fatigue from just static data is therefore still obviously attractive, since experience of static strength is so much easier and cheaper to obtain. Therefore, it is surprising to read in [3] that Expressions for the cycles to failure as a function of $R$ ratio are derived. These expressions do not require any curve fitting and do not involve any experimentally determined parameters. The fatigue predictions do not require any fatigue tests for calibration". Further, that comparison to several test cases found in the Literature show this first simple model to be very promising", where for several test cases", Ref. [3] intends a few references (Ref. [34-41] which are [7-14] here), where the error is said to be small but which in fact is not necessarily so. Take Fig.6 of Kassapoglou's paper [3], where the agreement is said to be very good": Ref.[3]'s curve, which should be the median value, is seen to pass close to the lowest data, and hence the error in terms of life can be easily of 2 orders of magnitude. Not much better can be said regarding Fig.9 (where the author admits the agreement to be "not so good"): the author prefers to measure the error based on stress, and claims a 17\% error is found - clearly, the error in terms of life can be of various orders of magnitude. Similar problems were found in Figs. 10 and 11, where the data are so few that do not really permit many conclusions to be drawn. Therefore, the sentence in the conclusion The approach allows analytical determination of the ratio of mean to Bor A-basis life which can be used in designing certification of qualification programs" seems premature. The data show that the link with static scatter is not so strong as to make any remote estimates of the $\mathrm{SN}$ curve slopes.

Ref. [3,4,5] seem to have followed an indication in Ref. [8] which at page 527 refers to the fact that many composite Wohler diagram (SN curves) seem to have a life distribution with Weibull shape parameter close to 1 (exponential distribution), writing "The exponential distribution is sometimes used to model distributions of failures times for the reliability of a product. It is pointed out by Chatfield that the exponential distribution governs systems where age has no influence on the probability of failure"... "This would not normally be thought applicable to the fatigue failure of reinforced plastic, for which it is known that residual performance is in fact reduced as a result of the accumulation of damage". Ref. [4, 5], however, seem to confirm this result even in the counterintuitive case of wearout, by adopting a special wearout law. We shall show that this is contrived result, as confirmed by a few recent attempts in the Literature to adopt this model [15-17]. We shall draw attention in this paper to the fact that we do not expect theoretically any reason for a good predictive capability to be realistic in general. 
The tendency to exponential distribution of fatigue lives is not very strong in general anyway, since in many large databases a full distribution of Weibull shape parameters is found $[8,18]$.

Fleck Kang and Ashby [18], in an authoritative review which contains also data on composite materials, produce a large set of maps covering a huge number of references, and in particular show in Fig. 5 (Fig.1, here reproduced with permission) the well-known fact that endurance limit $\sigma_{e}$ scales in a roughly linear way with yield strength, $\sigma_{y}$. The fatigue ratio, defined as $\sigma_{e} / \sigma_{y}$ (but more classically for metals, $\sigma_{e} / \sigma_{f s}$ ) at load ratio $R=-1$, appears as a set of diagonal contours. The value of fatigue ratio, for engineering materials, usually lies between 0.3 and 1. Generally speaking, it is near 1 for monolithic ceramics, about 0.5 for metals and elastomers, and about 0.3 for polymers, foams and wood; the values for composites vary more widely--from 0.1 to 0.5 . Naturally, for fatigue limit in composites (as well as light alloys), often intended is the value at a given fixed number of cycles. This wide variation already makes one wonder that for composites the fatigue properties depend less on static properties than on other materials. Fleck Kang and Ashby [17] remark The wide range of fatigue ratios shown by composites relates, in part, to the wide spectrum of materials used to make them, and to the necessarily broad definition of failure: in particulate composites, failure means fracture; in fibrous composites it means major loss of stiffness.

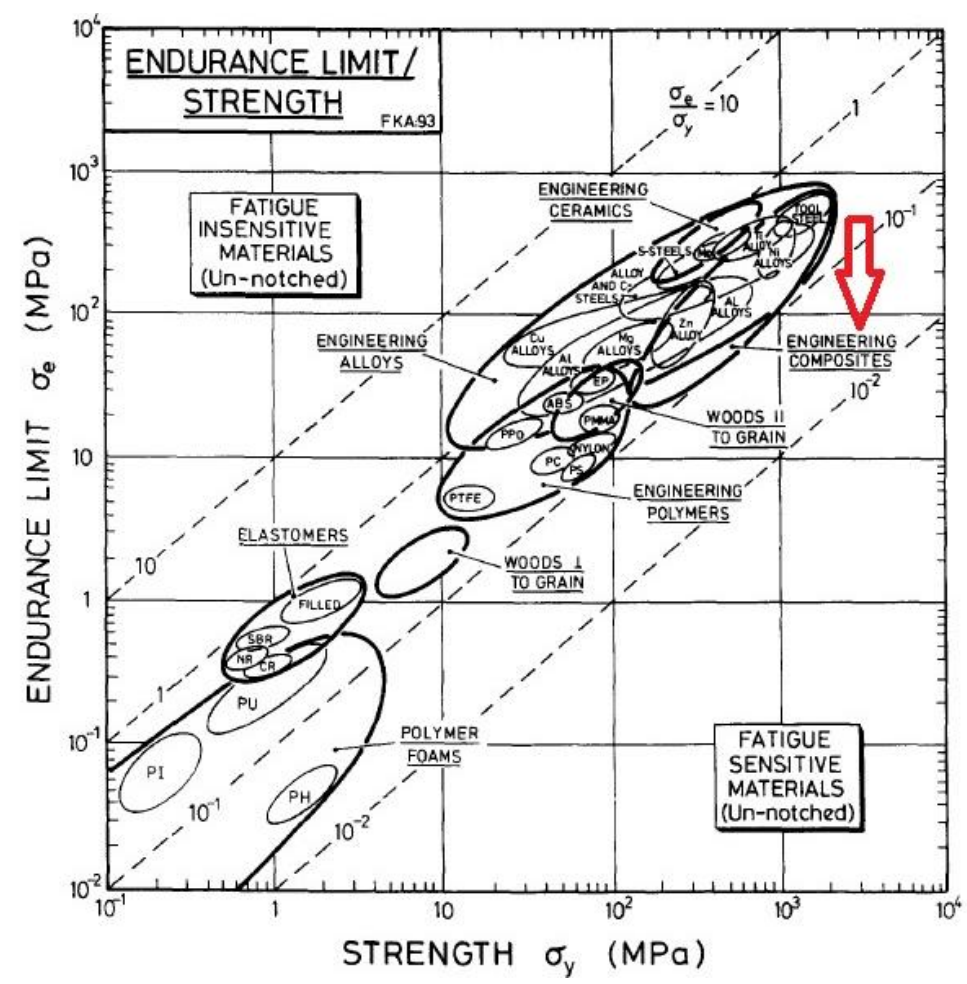

Fig. 1 From Fleck Kang and Ashby [18] (with permission).

Classical plot of static vs. fatigue strength for many classes of materials 
Clearly, the exact mechanisms for fatigue limit, if there is one, are microscopic and they, however, may interact with geometry, loading conditions, etc. For composite materials and structures in general, the failure mechanism can vary. For materials for which the endurance limit depends on the formation of slip bands, it is obvious to find a correlation with yield strength, but a full microscopic model for the shape of the SN curve is more difficult. Fleck et al [18] summarize about SN curve: It is the failure envelope associated with a sequence of interdependent phenomena: cyclic hardening, crack nucleation and cyclic growth, and final fast fracture. For composites, the actual nature of each phenomenon is very different but their interdependence is also clear. Kassapoglou's model is based on some statistical reasoning over the distribution of static strength, and the successive application of cycles. Making a certain number of (reasonable) assumptions, he seems to derive apparently simple and clear results, which he then combines with a calculation of probability of failure. We shall discuss in the present note the basic results of Kassapoglou's model in details.

\section{MAIN ASSUMPTIONS IN KASSAPOGLOU's MODEL}

In Ref. [3], Kassapoglou makes a certain number of assumptions, including that the probability of failure stays constant, cycle-by-cycle. Since the probability of failure during the first cycle is determined by a probability distribution function for the static strength, the author concludes that it remains the same for all subsequent cycles. In the later paper [4], this was obtained rigorously. In fact, even if the probability of failure did remain constant cycle-per-cycle, this is incorrect calculation for a fatigue experiment. That reliability theory permits "failure rate" to be obtained this way does not correspond to a SN curve.

As an illustrative example of these incorrect calculations let us consider the following discrete analogue:

1) There is a bucket full of balls that are numbered with 1 (which is analogous to failure of the specimen under a certain applied stress, "success") and 0 (unsuccessful event, no failure of the specimen). Assume that the probability of failure is $p$, so that the probability of no-success is $q=1-p$.

2) One can perform a series of experiments counting number $k$ of trials until the picked ball has a number 1 (success) on it. This event is called first success. After each attempt, in order to implement an analogue of the main assumption of the Kassapoglou's model, one must put the picked ball back into the bucket: then, and only then the probability of success is exactly the same at each single trial. The resultant distribution of number of trials until first success is described by the geometric distribution density function: probability $p_{1}$ that the first success" occurs at the $k$ th trial (cycle) is

$$
p_{1}(k)=p(1-p) .
$$

3) Eq. (probability first success) is simply the probability of success (failure) at last attempt (cycle) $k$ times the probability of no-failure at previous $k-1$ attempts (cycles). The average expected number $\langle\mathrm{n}\rangle$ of trials until the first success" is then given by 


$$
\langle n\rangle=\sum_{k=1}^{+\infty} k p(1-p)^{k-1}=\frac{1}{p},
$$

4) as indeed should be expected.

5) We can also calculate the probability that the failure of the sample occurs during the first $n$ experiments (cycles), i.e. that the event "first success" happens at any cycle $k$ in between 1 and $n$. This probability is the cumulative distribution of

$$
P(n)=\sum_{k=1}^{n} p_{1}(k)=\sum_{k=1}^{n} p_{1}(1-p)^{k-1}=1-(1-p)^{n}=1-\exp \left(-n \log \frac{1}{1-p}\right) .
$$

6) If now the number of trials $n$ is treated as a continuous variable, this function represents the so-called cumulative exponential distribution with the mean value

$$
\langle n\rangle=\frac{1}{\log \frac{1}{1-p}}=-\frac{1}{\log (1-p)},
$$

7) and if $p$ is small, the two averages (average number of trials) and (exponential average) become close.

This trivial example implements the main assumption of Kassapoglou's model. However, a randomly picked specimen is tested each time; each test is an independent static test with the probability of failure $p$ being equal to the probability that the static strength of the specimen is less than applied static stress $\sigma$. Obviously, this type of test is irrelevant to the fatigue phenomenon. The resulting relation between the mean number of cycles and the applied load, mistakenly claimed by the author to be a SN curve, is actually the mean number of tested specimens until failure! If the same specimen undergoes subsequent loadings, it may fail only if its static strength decreases with cycles. However, this static strength degradation should be a material specific function and is not uniquely determined by the statistics of the static strength. While dispersion in the static strength of a material reflects the possible level of initial damage observed in the material, the fatigue failure phenomenon is the result of the initial damage growth and accumulation with cyclic loading. This growth would eventually appear in any specimen independently of the strength in other specimens and the statistics that describes the static strength scatter.

\section{KASSAPOGLOU'S MODEL WITH STRENGTH DEGRADATION}

In his $2012 \mathrm{PhD}$ thesis [5] and in his 2011 paper [4] Kassapoglou extends the model and incorporates the residual strength degradation. Suppose that a constant amplitude load with maximum stress $\sigma\left(R=\sigma_{\min } / \sigma=0\right)$ is applied to a composite structure. If static failure strength $\sigma_{f s}$ of this structure is less or equal to $\sigma$ (i.e. $\sigma_{f s} \leq \sigma$ ) the structure will fail at $n=0$, i.e. before the first cycle is completed, whilst if $\sigma_{f s}>\sigma$ the structure will fail at the cycle $n=N$. Within the Kassapoglou's wear-out model quantity $N$ is treated as constant and no failure can occur for $0<n<N$. These assumptions are, as we show in the sequel, the most critical flaws of Kassapoglou's model. 
If the test had stopped at any cycle level $n<N$ the structure would not have failed and it would still be able to carry load. However, a strength test on the structure would show a failure strength $\sigma_{f s}>\sigma_{r}>\sigma$, where $\sigma_{r}$ is the residual strength. Hence, during cycling, $\sigma_{r}$ decreases from the static failure strength $\sigma_{f s}>\sigma$ at $n=0$, to $\sigma$ after $N$ cycles. Kassapoglou's model starts with the assumption that the change in residual strength is proportional to the current residual strength, which in the simple case of zero fatigue limit can be written in the form

$$
\frac{d \sigma_{r}}{d n}=-A \sigma_{r}
$$

where $A>0$ is independent of $n$ and $\sigma_{r}$. This wear-out model assumes that under a fixed amplitude the strength of a specimen with higher residual strength stress will degrade faster than one with lower residual strength, which is physically unreasonable. That is why a typical wear-out model would define the strength degradation rate as a reciprocal to the current residual strength. The above expression in Kassapoglou's model results in a residual strength

$$
\sigma_{r}=\sigma_{f s}\left(\frac{\sigma}{\sigma_{f s}}\right)^{n /(N-1)} .
$$

Treating $\sigma$ as a constant would results, following Kassapoglou's arguments, in a Weibull cumulative distribution $P_{W}\left(\sigma_{r} ; \beta_{r}, \alpha_{r}\right)$ for residual strength with shape and scale parameters

$$
\alpha_{r}=\alpha \frac{N-1}{N-n-1} ; \quad \beta_{r}=\sigma^{n /(N-1)} \beta^{\frac{N-n-1}{N-1}}
$$

which vary with $n$ in a simple manner, implying a clear reduction in experimental scatter with a lower stress level of testing (or longer lives). In particular, for $n \rightarrow N-1, \alpha_{r} \rightarrow \infty$ which means that the distribution converges to the Dirac Delta function, the residual strength becomes a deterministic function, and $\beta_{r}=\sigma$ consistently to the fact that the residual strength tends exactly to the SN curve.

There are many critical inconsistencies that one can spot immediately: Firstly, $N$ from the SN curve should itself be a variety, instead of being a deterministic and constant quantity. Secondly, with a constant amplitude $\sigma$, during cycling the residual strength distribution cannot approach the SN curve from below, since a specimen that has strength below $\sigma$ should have failed at an earlier stage and for any $n$ the residual strength distribution should be truncated from below by applied stress $\sigma$. One can even and easily show that, in contrast to what has been claimed by $K$, Kassapoglou's model cannot lead to a cycle-by-cycle constant probability of failure $p(n)$, which indeed, even within the hypothesis of Kassapoglou's wear out model, would be

$$
p(n)=\delta_{0 n} P_{W}(\sigma ; \beta, \alpha)+\delta_{n N}\left[1-P_{W}(\sigma ; \beta, \alpha)\right],
$$

where $\delta_{k h}$ is the Kronecker delta (see Appendix II). The discussed inconsistencies in the model development refute the claim of rigorous proof of constant cycle-by-cycle probability of failure. Here, it is instructive to cite that many authors in the Literature find 
distribution of residual strength which shows a decrease of Weibull's $\alpha$, rather than an increase as predicted by Kassapoglou.

\section{COMPARISON WITH EXPERIMENTAL DATA}

The original Kassapoglou's model did not find an exact Weibull distribution for the life, but over a very wide range of $p$ values ( $p<0.1$ hence, unless the applied load is very high, and close to the static strength) the two ratios of mean and modal lives to B-Basis life (respectively 17.86 and 8.93 ) are essentially constant. This suggested the author of the original Kassapoglou's model that the average of the two ratios, 13.4 is very close to the value of 13.6 determined in the NAVY reports [6] after statistical analysis of thousands of data points.

Using a slightly different re-derivation (see Appendix I), we show that Kassapoglou's approach essentially obtains an exponential distribution of fatigue lives, for a Weibull starting point in scatter of static data. Hence, it is correct to say that Kassapoglou's approach, in a slightly re-elaborated form (see Appendix I), we easily obtain the estimate on fatigue ratio (FR, defined as the ratio between fatigue limit at $10^{\wedge} 6$ cycle, and the static value" at $N=1$ ), as

$$
F R(\alpha)=\frac{\sigma_{\text {max,lim, } 10^{-6}}}{\sigma_{\max , 1}}=10^{(-6 / \alpha)},
$$

where $\alpha$ is a Weibull shape parameter in a 2 parameters Weibull distribution, of the static strength distributions. Notice that in this form, Kassapoglou's model includes also the Rratio effect, for $0<R<1$ ie in the cases of pure tensile loading. For $\alpha$ we can make use not of few sparse references like $\mathrm{K}$, but the thousands of test done by the Navy. The distribution of fatigue lives is not as unique as to be an exponential $\alpha_{L}=1$. A full distribution was found, which we may define as the distribution of scatter of fatigue lives, $\alpha_{L}$. A generally accepted approximation is to take $\alpha_{L}=1.25$, but it is clear that its distribution is relatively wide, and depends also on the method used for analysis.

Also, the distribution of static strength scatter $\alpha$ has in turn a distribution, which we can denominate $\alpha_{a}$, see Fig. 2.

We can use the mean value of $\alpha$ which results from the Fig. 2 to be 26. This corresponds to a value

$$
F R\left(\alpha_{\text {mean }}\right)=10^{(-6 / 26)}=0.5878,
$$

which is outside the known values of Ashby fatigue ratios. This suggests, as confirmed by most experimental data we shall describe, that Kassapoglou's method would tend to give unconservative estimates, as too high FR. This, however, depends very much on the type of materials under examinations. If the materials are of "poor" quality, full of defects, tending to having low $\alpha$, then the very steep SN curve predicted by K may neglect the possible phase in $\mathrm{SN}$ curve where the degradation is not so evident, resulting as extremely conservative. On the other hand, for materials having very high $\alpha$ like close to a metal, Kassapoglou's theory fails to capture the wearout at all, and results in too optimistic SN curve. Unidirectional laminates will tend to have very horizontal fatigue lines, yet their static scatter may be significant. 

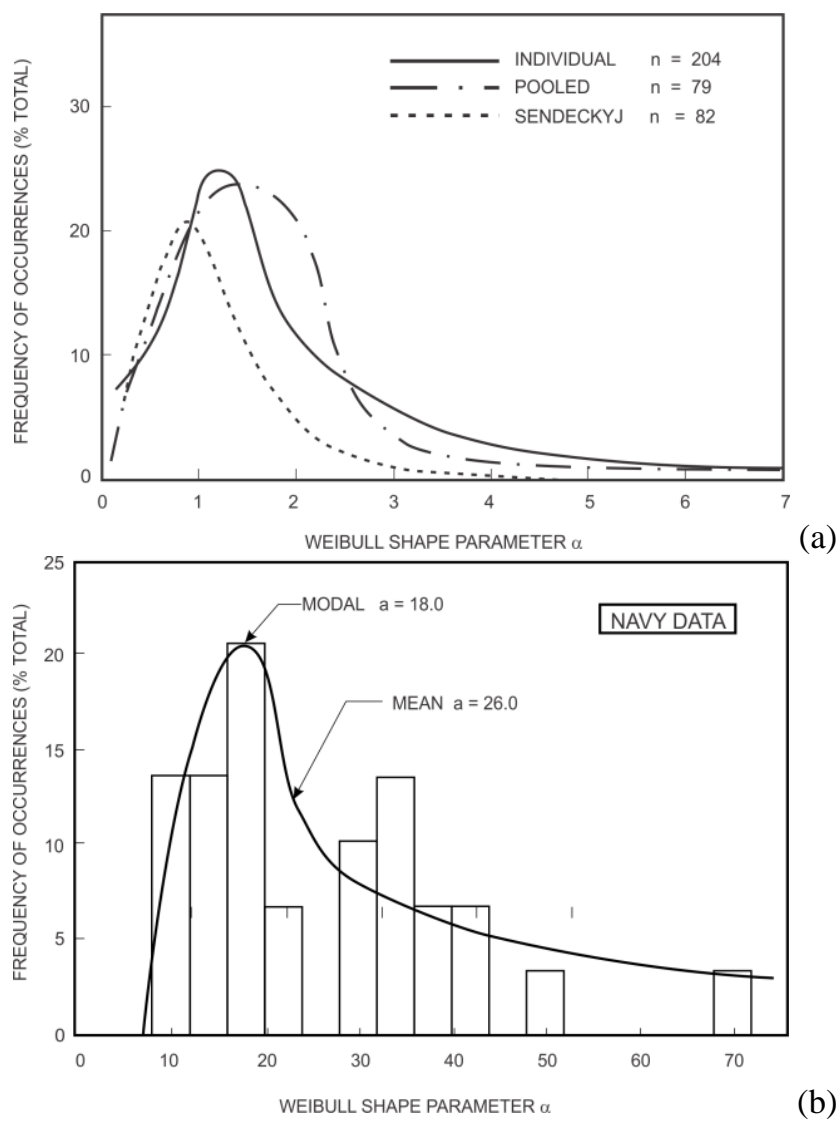

Fig. 2 (a) Distribution of fatigue lives scatter $\alpha_{L}$ and (b) of scatter of static strength $\alpha$

Hence even if the agreement with Navy experiments has some very loose qualitative agreement in terms of scatter of fatigue lives, this is an oversimplification (a single mode value instead of the full distribution) and the huge risks of using this approximation even as a crude estimate is evident.

The recent investigation of Kassapoglou's method by the FAA (Tomblin and Seneviratne, 2011 [5], Appendix A) finds also the SN curve predicted by the original Kassapoglou's model (which we found here as the mean life curve) to have rather erratic comparison with experimental data. In particular, in 14 sets of data, Kassapoglou's model was found

- accurate only for 2 sets,

- conservative only for 2 sets (both by 1-2 orders of magnitude),

- unconservative for the vast majority of data (10 sets), of which 5 perhaps by 1-2 orders of magnitude, 3 by 2-3 orders of magnitude, and 2 by 4-5 orders of magnitude.

Clearly, although the statistics does not say much, it shows a tendency of Kassapoglou's method to overpredict fatigue life by large factors. Examples given in Chapt. 6 of [3] and in [19] show that more sophisticated methods with variable $p$ function may improve the 
situation, although the examples given tend to predict longer lives than the original $\mathrm{K}$ method --- we are not able to judge if the errors and approximations from the original $\mathrm{K}$ theory with "constant $p$ value" continue to manifest their negative effect here. S/N curves based on the Sendeckyj analysis [2] were found generally accurate and conservative, but this is to be expected since that method is a fitting method of SN data.

In a recent book, Vassilopoulos \& Keller [16] compare 4 methods to make a statistical analysis of fatigue data, which is a problem of enormous industrial interest since aeronautical structures are designed and certified using SN curves that correspond to high reliability levels in the range above $90 \%$ and conform with design codes, but without an impossibly expensive program of fatigue testing on a population of full scale structures. The method based on the normal lifetime distribution (NLD) was found as nonconservative, giving a median SN curve which is closer to the median SN curve of ASTM than the 95\% reliability one. Whitney's pooling scheme and Sendeckyj's wear-out model are found to produce similar SN curves, with Whitney's easier to implement, as not requiring any optimization process, and Sendeckyi being also less conservative. However, this is mainly due to the need of multiple fatigue results at each stress level, and no capability to consider static data. Some significant problems were found in the fitting of Sendeckyi's constant process, with strange slopes of the SN curve predicted, particularly when disregarding static strength data. A discussion follows on the appropriateness of including the static data in the fitting.

Kassapoglou's method is not even compared to the previous four, mainly because the static data were not enough to fit Weibull distributions. It is discussed, however, in its extension to describe mean stress effect, in a later chapter on Constant Life Diagrams. However, its assumptions are negatively judged This assumption oversimplifies the reality and masks the effect of the different damage mechanisms that develop under static loading and at different stages of fatigue loading, and the restricted use of static data disregards the different damage mechanisms that develops during fatigue loading and in many cases leads to erroneous results. In the evaluation of Kassapoglou's model for one database the model proved to be inaccurate for the examined material's fatigue data.

\section{CONCLUSIONS}

In the original Kassapoglou's model there is confusion between what we call fatigue and statistics of the static strength of a number of specimens, which stems from an incorrect reference to the reliability theory of failure rate of products. Fatigue life (number of cycles) is mistakenly replaced with the number of tested specimens to find a specimen with strength less than the applied load. This number of specimen indeed solely depends on the initial statistical distribution of the static strength, while fatigue is related to damage accumulation in a specimen and its strength degradation with cycles, which contradicts the main assumption. One can also mistakenly deduce from the proposed model that if there is no dispersion in the static strength, for instance, all the specimen have exactly the same static strength, there is no such thing as an SN curve. Kassapoglou's model is an interesting attempt of using wear-out models with degradation deterministic equations to predicting SN curves from static data only for composites (something which is not easy even with metals). However, its results do not look realistic at all, and indeed 
we have here explained why. Not surprisingly, SN curves found in many independent assessments were found to be (generally) unconservative for the vast majority of data (10 sets) considered in FAA 2011 report [15], at least by 1-2 orders of magnitude. We have given reasons for this effect, both theoretically and with additional estimates from large set of results from databases of composite materials.

Only "fitting" models can be considered reliable, as discussed by Vassilopoulos \& Keller [16], and it should be remarked in this respect that an additional interesting wearout model is [21-23].

\section{REFERENCES}

1. Hahn, H.T., Kim, R.Y., 1975, Proof testing of composite materials. J Composite Materials, 9, pp. $297-311$.

2. Sendeckyj, G.P., 1981, Fitting models to composite materials fatigue data. test methods and design allowables for fibrous composites, In: Chamis, C.C. (ed), ASTM STP 734. Philadelphia, PA: American Society for Testing and Materials, pp. 245-260.

3. Kassapoglou, C., 2007, Fatigue life prediction of composite structures under constant amplitude loading, J of Composite Materials, 41, pp. 2737-2754.

4. Kassapoglou, C., 2011, fatigue model for composites based on the cycle-by-cycle probability of failure: implications and applications, J of Composite Materials, 45, pp. 261-277.

5. Kassapoglou, C., 2012, Predicting the structural performance of composite structures under cyclic loading, $\mathrm{PhD}$ Thesis, Delft Univ of Technology, Netherlands

6. Juvinall, R.C., Marshek, K.M., 2011, Fundamentals of machine component design, 5th ed. John Wiley \& Sons Inc, USA.

7. Lee, J-W., Daniel, I.M., Yaniv, G., 1989, Fatigue life prediction of cross-ply composite laminates. In: Lagace, P.A. (ed), Composite Materials: Fatigue and Fracture, Second Volume. ASTM STP 1012, Philadelphia, PA: American Society for Testing and Materials, pp. 19-28.

8. Gathercole, N., Reiter, H., Adam, T., Harris, B., 1994, Life prediction for fatigue of T800/5245 carbon-fibre composites: I. Constant amplitude loading, Fatigue, 16, pp. 523-532.

9. Amijima, S., Fujii, T., Hamaguchi, M., 1991, Static and fatigue tests of a woven glass fabric composite under biaxial tension-torsion, Composites, 22, pp. 281-289.

10. Cvitkovich, M.K., O'Brien, T.K., Minguet, P.J., 1998, Fatigue debonding characterization in composite skin/stringer configurations, In: Cucinell, R.B. (ed.), ASTM STP 1330, Philadelphia, PA: American Society for Testing and Materials, pp. 97--121.

11. O'Brien, T.K., 1988, Fatigue delamination behavior of peek thermoplastic composite laminates, J. Reinforced Plastics and Composites, 7, pp. 341-359.

12. O'Brien, T.K., Rigamonti, M., Zanotti, C., 1988, Tension fatigue analysis and life prediction for composite laminates, Hampton, VA: National Aeronautics and Space Administration. Technical Memorandum 100549

13. Maier, G., Ott, H, Protzner, A., Protz, B., 1986, Damage development in carbon fibre-reinforced polyimides in fatigue loading as a function of stress ratio, Composites, 17, pp. 111--120.

14. Gerharz, J.J., Rott, D., Schuetz, D., 1979, Schwingfestigkeitsuntersuchungen an Fuegungen in Faserbauweise, BMVg-FBWT, pp. 79-23.

15. Tomblin, J., Seneviratne, W., 2011, Determining the fatigue life of composite aircraft structures using life and load-enhancement factors, Report DOT/FAA/AR-10/6. Federal Aviation Administration, National Technical Information Service, Springfield, USA, www.tc.faa.gov/its/worldpac/techrpt/ar10-6.pdf (last access: 12.03.2018)

16. Vassilopoulos, A.P., Keller, T., 2011, Fatigue of fiber-reinforced composites, London: Springer-Verlag, UK

17. Andersons, J., Paramonov, Yu., 2011, Applicability of empirical models for evaluation of stress ratio effect on the dura.bility of fiber-reinforced creep rupture-susceptible composites, J Mater Sci, 46, pp. 1705-1713.

18. Fleck, N.A, Kang, K.J., Ashby, M.F., 1994, Overview no. 112: The cyclic properties of engineering materials, Acta Metallurgica et Materialia, 42, pp. 365-381.

19. Whitehead, R.S., Kan, H.P., Cordero, R., Saether, E.S., 1986, Certification Testing Methodology for Composite Structures, Vol I and II. Naval Air Development Centre Report No. 87042-60 (DOT/FAA/CT-86-39), http://www.dtic.mil/dtic/tr/fulltext/u2/b112288.pdf (last access: 15.03.2018)

20. Kassapoglou, C., Kaminski, M., 2011, Modeling damage and load redistribution in composites under tensiontension fatigue loading, Composites: A, 42, pp. 1783-1792. 
21. D'Amore, A., Caprino, G., Stupak, P., Zhou, J., Nicolais, L., 1996, Effect of stress ratio on the flexural fatigue behaviour of continuous strand mat reinforced plastics, Science and Engineering of Composite Materials, 5, pp. 1-8.

22. Caprino, G., D'Amore, A., 1998, Flexural fatigue behaviour of random continuous fibre reinforced thermoplastic composites, Composite Science and Technology, 58, pp. 957-965.

23. D'Amore, A., Caprino, G., Nicolais, L., Marino, G., 1999, Long-term behaviour of PEI and PEI-based composites subjected to physical aging. Composites Science and Technology, 59, 1993-200.

24. Post, N.L., 2005, Modeling the Residual Strength Distribution of Structural GFRP Composite Materials Subjected to Constant and Variable Amplitude Tension-Tension Fatigue Loading, PhD Thesis, University of Virginia. Blacksburg, Virginia, USA

\section{APPENDIX I - SN CURVE OF KASSAPOGLOU'S MODEL IN A SLIGHTLY DIFFERENT FORM}

We have shown that Kassapoglou's model is incorrect. However, a simpler form can be adapted for comparative form in a much simpler form. In particular, using this equation for a $\mathrm{SN}$ curve at any quantile $Q$

$$
N=\left(\frac{\beta}{\sigma}\right)^{\alpha}[-\log (1-Q)],
$$

which obviously has mean value $N_{m}=(\beta / \sigma)^{\alpha}$ and modal value $N_{\text {mod }}=(\beta / \sigma)^{\alpha} \log 2$, but mode value zero (because the distribution of lives is an exponential distribution $\alpha_{L}=1$ ), we obtain a closed form version of the "incorrect" $\mathrm{K}$ model, which can be used more easily than the original Kassapoglou's model which is not in closed form, and which obtains only the mode life $N_{c}$

$$
N_{c}=\left(\frac{\beta}{\sigma}\right)^{\alpha}
$$

which in the present result, coincides with the present mean value. The distribution in terms of stress for given number of cycles, $P_{W}\left(\sigma ; \beta / N^{l / \alpha}, \alpha\right)$

$$
\sigma=\left(\frac{\beta}{N^{1 / \alpha}}\right)[-\log (1-Q)]^{1 / \alpha} ;
$$

this has obviously mean value $\sigma_{m}=\left(\beta / \mathrm{N}^{1 / \alpha}\right) \Gamma(1+1 / \alpha)$, whereas median value $\sigma_{\text {med }}=$ $\left(\beta / \mathrm{N}^{1 / \alpha}\right)(\log 2)^{1 / \alpha}$. In other words, in this new form, the SN derives from Weibull distribution both in terms of stress and life at all levels of stress including the original static distribution $P_{W}(\sigma ; \beta, \alpha)$ which is obtained consistently for $N^{1 / \alpha}=1$.

\section{APPENDIX II - INCONSISTENCIES IN THE WEAR OUT MODEL OF REF. [4, 5]}

Suppose we want to calculate the cumulative probability distribution of failure $P(n)$ during the first $n$ cycles assuming Kassapoglou's wear-out model

$$
\frac{\sigma_{r}}{\sigma_{f s}}=\left(\frac{\sigma}{\sigma_{f s}}\right)^{n /(N-1)},
$$


where the $\sigma_{r}$ is the residual strength, $\sigma_{f s}$ is the static strength, $\sigma$ is the applied fatigue load, $n$ is the actual cycle number, and $N$ is the number of cycle at which the samples fails (assuming its static strength is larger than $\sigma$ ). Equation (wear out model) simply states that: (i) all sample which have a static strength $\sigma_{f s}$ larger than the fatigue stress $\sigma$ will fail at the same given number of cycles $n=N$, and (ii) samples with static strength $\sigma_{f s}$ less than $\sigma$ will all fail at cycle $n=0$. Therefore samples may fail either at $n=0$ when $\sigma_{s f} \leq \sigma$ or at $n=N$ when $\sigma_{s f} \geq \sigma$, no failure may occur in between i.e. for $0<n<N$. Within the Kassapoglou's wear-out model we have: (a) the probability that failure occurs at $n=0$ is $p_{0}=P_{W}(\sigma ; \beta, \alpha)$, (b) the probability that failure occurs at $n=N$ is $p_{N}=P\left(\sigma_{S}>\sigma\right)=P_{W}\left(\sigma_{2} \beta, \alpha\right)$, (c) the probability that failure occurs at $n$ satisfying the condition $0<n<N$ is $p_{n}=0$.

Now let us calculate the cumulative probability distribution of $P_{n}=\sum_{k=0}^{n} p_{k}$ with $n \geq 0$, which is the probability that the sample fails within the first $n \geq 0$ cycles, i.e. $P_{n}=P(0 \leq k<n)$. Since failure cannot occur in between 0 and $N$ (i.e. $p_{k}=0$ for $0<k<N$ ) the probability that failure occurs within the firs $n<N$ must be equal to the probability that failure occurs at cycle 0 , i.e.

$$
P_{n}=p_{0}=P_{W}(\sigma ; \beta, \alpha) ; \quad 0 \leq n<N,
$$

whereas considering that for $n \geq N$ failures has necessarily occurred one as

$$
P_{n}=1 ; \quad n \geq N .
$$

Therefore the cumulative distribution presents two steps one of amplitude $P_{W}(\sigma)$ at $n=0$ ant the other of amplitude $1-P_{W}(\sigma ; \beta, \alpha)$ at $n=N$. In between the cumulative probability distribution is constant. We stress that, as already shown, the probability of failure cycle per cycle is not constant indeed it is:

in compact notation

$$
\begin{aligned}
& p_{n}=P_{W}(\sigma ; \beta, \alpha) ; \quad n=N, \\
& p_{n}=0 ; \quad 0<n<N, \\
& p_{n}=1-P_{W}(\sigma ; \beta, \alpha) ; \quad n=N, \\
& p_{n}=0 ; \quad n>N,
\end{aligned}
$$

$$
p(n)=\delta_{0 n} P_{W}(\sigma ; \beta, \alpha)+\delta_{n N}\left[1-P_{W}(\sigma ; \beta, \alpha)\right],
$$

where $\delta_{j k}$ is Kronecker's delta. Eq. (probability cycle per cycle) shows that the probability of failure cycle per cycle is zero for $0<n<N$, thus revealing one of the serious mistakes of the Kassapoglou model where the cycle per cycle probability of failure was assumed different from zero and equal to $P_{W}(\sigma ; \beta, \alpha)$. Indeed, Eq. (probability cycle per cycle) shows that the sample life $n$ is a discrete statistical quantity which only takes two different values $n=0$ and $n=N$, and failure at $n=0$ occurs with probability $P_{W}(\sigma ; \beta, \alpha)$ whereas failure at $n=N$ occurs with probability $1-P_{W}(\sigma ; \beta, \alpha)$. This allows to calculate within the wear-out model (wear out model) the expected life of the samples as

$$
\langle n\rangle=N\left[1-P_{W}(\sigma ; \beta, \alpha)\right] .
$$

which as expected differs from the value obtained by K. 